Canad. Math. Bull. Vol. 21 (1), 1978

\title{
MULTIPLICITIES IN THE TENSOR PRODUCT OF FINITE-DIMENSIONAL REPRESENTATIONS OF DISCRETE GROUPS
}

\author{
DRAGOMIR ̌̌ Ž. DJOKOVIĆ
}

Let $G$ be a group and $\rho$ and $\sigma$ two irreducible unitary representations of $G$ in complex Hilbert spaces and assume that $\operatorname{dim} \rho=n<\infty$. D. Poguntke [2] proved that $\rho \otimes \sigma$ is a sum of at most $n^{2}$ irreducible subrepresentations. The case when $\operatorname{dim} \sigma$ is also finite he attributed to $\mathrm{R}$. Howe.

We shall prove analogous results for arbitrary finite-dimensional representations, not necessarily unitary. Thus let $F$ be an algebraically closed field of characteristic 0 . We shall use the language of modules and we postulate that all our modules are finite-dimensional as $F$-vector spaces. The field $F$ itself will be considered as a trivial $G$-module.

If $V$ and $W$ are $G$-modules then $\operatorname{Hom}_{F}(V, W)$ is also a $G$-module. If $f: V \rightarrow W$ is a linear map and $a \in G$ then $a \cdot f$ is the linear map $V \rightarrow W$ defined by $(a \cdot f)(v)=a \cdot f\left(a^{-1} \cdot v\right)$. In particular, when $W=F$ we get a $G$ module $V^{*}=\operatorname{Hom}_{F}(V, F)$.

If $V$ is a $G$-module then we denote by $V^{G}$ the submodule of $V$ consisting of $G$-invariant elements, i.e., elements $v \in V$ such that $a \cdot v=v$ for all $a \in G$. Note that if $V$ and $W$ are $G$-modules then

$$
\operatorname{Hom}_{G}(V, W)=\left(\operatorname{Hom}_{F}(V, W)\right)^{G} \text {. }
$$

If $V$ and $W$ are $G$-modules then we define

$$
\langle V, W\rangle=\operatorname{dim}_{F} \operatorname{Hom}_{G}(V, W),
$$

which is an integer usually called the intertwining number. It is clear that

$$
\begin{aligned}
& \left\langle V, W_{1} \oplus W_{2}\right\rangle=\left\langle V, W_{1}\right\rangle+\left\langle V, W_{2}\right\rangle, \\
& \left\langle V_{1} \oplus V_{2}, W\right\rangle=\left\langle V_{1}, W\right\rangle+\left\langle V_{2}, W\right\rangle .
\end{aligned}
$$

Let $V_{1}, V_{2}, V_{3}$ be finite-dimensional $F$-vector spaces. Recall that there exist canonical vector space isomorphisms

and

$$
V_{2}^{*} \otimes V_{3} \rightarrow \operatorname{Hom}_{F}\left(V_{2}, V_{3}\right)
$$

$$
\operatorname{Hom}_{F}\left(V_{1} \otimes V_{2}, V_{3}\right) \rightarrow \operatorname{Hom}_{F}\left(V_{1}, \operatorname{Hom}_{F}\left(V_{2}, V_{3}\right)\right)
$$

Received by the editors March 28, 1977.

This work was supported in part by NRC Grant A-5285. 
and consequently a canonical isomorphism

$$
\operatorname{Hom}_{F}\left(V_{1} \otimes V_{2}, V_{3}\right) \rightarrow \operatorname{Hom}_{F}\left(V_{1}, V_{2}^{*} \otimes V_{3}\right) .
$$

If $V_{1}, V_{2}, V_{3}$ are all $G$-modules then these canonical maps are also $G$ homomorphisms. Hence, it follows that the isomorphism (1) induces a vector space isomorphism

$$
\operatorname{Hom}_{G}\left(V_{1} \otimes V_{2}, V_{3}\right) \rightarrow \operatorname{Hom}_{G}\left(V_{1}, V_{2}^{*} \otimes V_{3}\right) .
$$

Therefore we have

$$
\left\langle V_{1} \otimes V_{2}, V_{3}\right\rangle=\left\langle V_{1}, V_{2}^{*} \otimes V_{3}\right\rangle .
$$

Taking $V_{1}=F$ and writing $V_{2}=V, V_{3}=W$, we obtain

$$
\langle V, W\rangle=\operatorname{dim}_{F}\left(V^{*} \otimes W\right)^{G} .
$$

If $V$ is a simple $G$-module and $W$ a semi-simple $G$-module then $\langle V, W\rangle$ is the multiplicity of $V$ in $W$. This follows from the fact that $\operatorname{End}_{G}(V)=F, F$ being algebraically closed. If both $V$ and $W$ are semi-simple $G$-modules then clearly $\langle V, W\rangle=\langle W, V\rangle$.

We recall that the tensor product of semi-simple $G$-modules is also semisimple because char $F=0$, see [1, p. 85].

If $V$ is a $G$-module and $n$ an integer $\geq 0$ then $n V$ denotes direct sum of $n$ copies of $V$.

Theorem 1. Let $U, V, W$ be simple $G$-modules and $m, n, k$ their dimensions. respectively. Assume that $m \leq n \leq k$. Then

$$
\operatorname{dim}_{F}(U \otimes V \otimes W)^{G} \leq m
$$

and equality holds if and only if $n=k$ and $U \otimes V \cong m W^{*}$

Proof. Recall that the dual of a simple $G$-module is also simple. Using (2) and (3) we obtain

$$
\begin{aligned}
\operatorname{dim}_{F}(U \otimes V \otimes W)^{G} & =\langle F, U \otimes V \otimes W\rangle \\
& =\left\langle W^{*}, U \otimes V\right\rangle \\
& \leq \frac{m n}{k} \leq m .
\end{aligned}
$$

The assertion about the equality sign is now obvious.

THeOREM 2. Let $U, V, W$ be simple $G$-modules and $m, n, k$ their dimensions, respectively. Then the multiplicity of $U$ in $V \otimes W$ is less than or equal $\min (m, n, k)$.

Proof. Using (2) we obtain

$$
\begin{aligned}
\langle U, V \otimes W\rangle & =\left\langle F, U^{*} \otimes V \otimes W\right\rangle \\
& =\operatorname{dim}_{F}\left(U^{*} \otimes V \otimes W\right)^{G}
\end{aligned}
$$


and we can apply Theorem 1 to get the assertion.

Theorem 3. Let $V$ and $W$ be simple $G$-modules and $n=\operatorname{dim} V$. Then

$$
\operatorname{dim}_{F} \operatorname{End}_{G}(V \otimes W) \leq n^{2} .
$$

In particular, the length of the $G$-module $V \otimes W$ is $\leq n^{2}$.

Proof. Since $V \otimes V^{*}$ is semi-simple [1, p. 85] we have a direct decomposition $V \otimes V^{*}=V_{1} \oplus \cdots \oplus V_{k}$ where $V_{i}$ are simple $G$-modules. Using (2), (3), and Theorem 2, we obtain

$$
\begin{aligned}
\operatorname{dim}_{F} \operatorname{End}_{G}(V \otimes W) & =\operatorname{dim}_{F} \operatorname{Hom}_{G}\left(F, V \otimes V^{*} \otimes W \otimes W^{*}\right) \\
& =\sum_{i=1}^{k} \operatorname{dim}_{F}\left(V_{i} \otimes W \otimes W^{*}\right)^{G} \\
& \leq \sum_{i=1}^{k} \operatorname{dim}_{F}\left(V_{i}\right)=n^{2}
\end{aligned}
$$

The second assertion follows from the first.

The bound $n^{2}$ is best possible as shown by an example in [2].

\section{REFERENCES}

1. G. Hochschild: Introduction to affine algebraic groups, Holden-Day, San Francisco 1971.

2. D. Poguntke: Decomposition of tensor products of irreducible unitary representations, Proc. Amer. Math-Soc. 52 (1975), 427-432.

\footnotetext{
Department of Pure Mathematics

UNIVERSITY OF WATERLOO

Waterloo, Ontario, Canada
} 